\title{
Minocycline induced arthritis associated with fever, livedo reticularis, and pANCA
}

\author{
O Elkayam, M Yaron, D Caspi
}

\begin{abstract}
Objective-to describe a novel iatrogenic immunological reaction produced by minocycline.

Case reports-The clinical course and laboratory results of three women who presented with similar rheumatological manifestations after a prolonged exposure to minocycline are described. All three presented a unique reaction manifested by fever, arthritis/arthralgia and livedo reticularis during treatment with minocycline for acne vulgaris. The clinical syndrome was associated with high titre of serum perinuclear anticytoplasmatic antibodies (p-ANCA) and antimyeloperoxidase antibody (anti-MPO). Symptoms resolved after stopping the drug and recurred promptly after rechallenge in all three patients.

Conclusions-Minocycline, which is widely used in the treatment of acne, often without adequate supervision, may induce arthritis and livedo vasculitis associated with anti-MPO.
\end{abstract}

(Ann Rheum Dis 1996;55:769-771)

Minocycline hydrochloride is a semisynthetic tetracycline used for a variety of infections, and for treatment of acne vulgaris. ${ }^{1}$ Several adverse effects have been reported, including nausea, blood eosinophilia, ${ }^{2}$ transient vestibular symptoms, ${ }^{3}$ photosensitivity, ${ }^{4}$ hyperpigmentation, ${ }^{5}$ rashes, ${ }^{6}$ fever, ${ }^{7}$ hypersensitivity pneumonitis, ${ }^{8}$ and hepatitis. ${ }^{9}$ Few rheumatic manifestations of toxicity have been reported. ${ }^{9-11}$

\section{Case reports}

CASE 1

Rheumatology, Ichilov

Hospital, Tel Aviv

Medical Centre, and

the Tel Aviv University

Sackler School of

Medicine, Israel

O Elkayam

M Yaron

D Caspi

Correspondence to: Ori Elkayam, Department of Rheumatology, Ichilov

Hospital, Tel Aviv Medical

Centre, Tel Aviv, Israel.

Accepted for publication 6 June 1996
A 19 year old woman presented a two month history of fever, under $38^{\circ} \mathrm{C}$ in the first seven weeks, rising to $39^{\circ} \mathrm{C}$ in a constant diurnal pattern the week preceding admission. She also complained of exhaustion and bilateral ankle pain. Her only past medical history was acne, treated with minocycline $100 \mathrm{mg}$ daily from the age of 15 to 18 . Three months before admission, she had renewed regular minocycline. On examination, she had fever of $39^{\circ} \mathrm{C}$, acrocyanosis of hands and feet, livedo reticularis of the legs, and mild swelling and exquisite tenderness of both ankles.
Laboratory investigations are reported in the table. Treatment with naproxen $500 \mathrm{mg}$ twice daily partially relieved her symptoms and rendered her afebrile. During the next 10 months-while taking minocycline intermittently-she suffered relapses which retrospectively could be related to her self administered courses. Her livedo reticularis worsened and a skin biopsy revealed perivascular inflammatory infiltrates. The patient herself raised the possibility of a connection between minocycline and her symptoms. Minocycline was discontinued with resolution of her fever within days. Two weeks later, the patient was rechallenged with a single tablet of $100 \mathrm{mg}$ minocycline. She developed fever up to $39^{\circ} \mathrm{C}$ and severe arthralgia within 24 hours, resolving completely after a few days. Although the patient has been asymptomatic ever since, serum p-ANCA remains positive 12 months later, but its titre is progressively decreasing (from $1 / 1280$ at the beginning of the disease to $1 / 160)$.

CASE 2

A 20 year old woman presented a six week history of fever, severe fatigue, myalgias, and arthralgia affecting shoulders, hands, and ankles. Her past medical history was unremarkable except for acne treated with minocycline from the age of 17 to 19 . Minocycline had been stopped for six months and renewed a few weeks before her current illness. On examination, the patient appeared ill with fever up to $39^{\circ} \mathrm{C}$, livedo reticularis on both legs, and symmetrical synovitis of wrists, meta-

Laboratory tests on admission

\begin{tabular}{llll}
\hline & Case 1 & Case 2 & Case 3 \\
\hline ESR & 90 & 60 & 40 \\
ANA & - & + & + \\
Anti-DNA & Normal & Normal & Normal \\
Anti-histone & - & - & + \\
Anti-Ro, La & - & - & - \\
Anti-cardiolipin & - & - & - \\
Lupus anticoagulant & - & - & - \\
C'3 C'4 & Normal & Normal & Normal \\
p-ANCA & $1 / 1280$ & $1 / 640$ & $1 / 1280$ \\
Anti-MPOa & + & + & + \\
Anti-elastase $^{b}$ & - & - & - \\
Anti-PR3 $^{\mathrm{a}}$ & - & - & - \\
\end{tabular}

ESR, erythrocyte sedimentaion rate; ANA, antinuclear antibodies; MPO, myeloperoxidase.

' ELISA, ELIAS-VAREIISA kit, code 19296.

b Clone system, EL-elastase kit, code 11-1100. 
carpophalangeal joints, proximal interphalangeal joints, and ankles. Laboratory tests are reported in the table. Stopping minocycline induced a complete resolution of fever and improvement in her arthralgias within days. Rechallenge with a single tablet of minocycline two weeks later provoked fever of $39^{\circ} \mathrm{C}$ and synovitis of both hands and ankles. Within 24 hours, fever resolved completely while arthralgia persisted. A two week course of treatment with prednisone, initially $15 \mathrm{mg} / \mathrm{d}$, induced complete resolution of symptoms. Nine months later, the patient remains asymptomatic with a slowly decreasing P-ANCA titre.

CASE 3

A 22 year old woman presented a three week history of fever, severe fatigue, myalgias, and pain in her elbows, ankles, and wrists. Since adolescence, she had suffered from acne and had taken minocycline from the age of 17 to 20. Minocycline was renewed a few weeks before her current symptoms.

On examination, she had fever of up to $38.5^{\circ} \mathrm{C}$, livedo reticularis on both legs, symmetrical synovitis of elbows and tenderness of both ankles. Laboratory tests are summarised in the table. Withdrawing minocycline induced complete resolution of fever within 24 hours and of the musculoskeletal symptoms within two weeks. Rechallenging with minocycline promptly reproduced fever, myalgia, and arthralgia within hours. This resolved after stopping the drug.

\section{Discussion}

We report three women with a common iatrogenic clinical picture of marked pyrexia, severe fatigue, polyarthritis, and livedo reticularis, associated with acute phase response and antiMPO in the serum. All had used minocycline for prolonged periods. Common to all three was renewal of the drug after a variable period (12-24 months) of withdrawal, and symptom onset within just a few weeks of restarting the drug. Symptoms resolved after stopping minocycline and recurred promptly after rechallenge, clearly implicating the drug in causation. The slow but persistent decline of anti-MPO autoantibodies during remission further supports a cause-effect relation. Although there are only three patients, it seems the more prolonged the exposure to minocycline after its renewal the more severe the clinical course.

A serum sickness-like syndrome with urticaria, fever, lymphadenopathy, and joint symptoms has been reported with minocycline. ${ }^{10}$ Polyarthritis with autoimmune hepatitis due to minocycline has also recently been reported. ${ }^{9}$ Minocycline has been associated with acute drug induced lupus syndrome with positive antinuclear antibodies in five young women taking minocycline for years. In four of these, clinical features disappeared shortly after stopping minocycline, whereas in one case the illness persisted and required treatment with corticosteroids. Resolution of serological abnormalities in these patients occurred more slowly than the clinical symptoms. ${ }^{11}$ Although our third patient presents some similarities to those cases (antinuclear and antihistone antibodies), our patients differ in having marked livedo reticularis, high titre p-ANCA, and absence of antinuclear antibodies and antihistones in two cases.

Autoantibodies against cytoplasmatic determinants of neutrophilic granulocytes are important diagnostic markers in systemic vasculitis. Circulating antibodies against myeloperoxidase, elastase, cathepsin G, lactoferrin, and lysozyme have been identified as causes of the p-ANCA phenomenon. ${ }^{12}$ Though MPO-ANCA have been detected in several non-vasculitic chronic inflammatory conditions like rheumatoid arthritis, juvenile rheumatoid arthritis, Felty syndrome, ulcerative colitis, and systemic lupus erythematosus (SLE), they are usually infrequent and at low titre. ${ }^{13}$ High serum levels of anti-MPO have been found in microscopic polyangiitis and Churg Strauss syndrome ${ }^{14}$ and in drug induced lupus caused by hydralazine and procainamide. ${ }^{15}{ }^{16}$ In all six patients with SLE-like syndrome induced by hydralazine treatment, anti-MPO autoantibodies were detected and antielastase in five of them. ${ }^{16}$ We believe that case 3 may indeed represent drug induced lupus in which p-ANCA is found at a very high level.

In addition to hydralazine and procainamide, propylthiouracil has been found to be associated with vasculitis, positive ANCA, and antibodies against human neutrophil elastase, proteinase 3 and myeloperoxidase in six patients. Following withdrawal of the drug, clinical symptoms resolved completely and ANCA concentrations decreased though they persisted even after one year of follow up. ${ }^{17}$

Crescentic glomerulonephritis induced by penicillamine, ${ }^{18}$ hydralazine, ${ }^{19}$ and carbimazole $^{20}$ associated with ANCA have also been reported. Likewise, interstitial nephritis due to omeprazole has been linked with serum p-ANCA. $^{21}$

All major classes of drugs that induce a lupus-like syndrome, such as hydralazine, procainamide, isoniazid, quinidine, chlorpromazine, and propylthiouracil, are possible substrates for myeloperoxidase, ${ }^{22}{ }^{23}$ their cytotoxic properties depending on the fact that neutrophil myeloperoxidase is implicated in the conversion of these drugs into toxic metabolites. ${ }^{24}$ Those data and the experience with hydralazine, procainamide, penicillamine, omeprazole, and antithyroid drugs, and the present report on minocycline, suggest that ANCA, beside being a useful marker in the detection of drug induced connective tissue disease, is involved in the pathogenesis of the iatrogenic disease.

We believe that minocycline may induce a characteristic vasculitic reaction manifested by fever, fatigue, arthritis/arthralgia, and livedo reticularis in the presence of positive immunofluorescent p-ANCA and anti-MPO. Minocycline is widely used by young female patients and awareness of this reaction is thus important. Prompt suspicion and discontinuation of minocycline will usually result in resolution. 
1 Rossman RE. Minocycline treatment of tetracycline resistant and tetracycline responsive acne vulgaris. Cutis ant and tetracyclin.

2 Sanole MA, Mandell GL. Antimicrobial agents: tetracyclines, chloramphenicol, erythromycin and miscellaneous antibacterial agents. In: Gilman AG, Rall TW, Nies AS, Taylor P, eds. Goodman and Gilman's The pharmacological basis of therapeutics, 8th ed. Elmsford NY: Pergamon Press, 1990:1117-46.

3 Allen JC. Minocycline. Ann Intern Med 1976;85:482-7.

4 Frost P, Weinstein GD, Gomez EC. Phototoxic potential of minocycline and doxycycline. Arch Dermatol 1972; 105:681-3.

5 Ridgway HA, Sonnex TS, Kennedy CTC, Millard PR, Henderson WJ, Gold SC. Hyperpigmentation associated with oral minocycline. Br f Dermatol 1982;107:95-102.

6 Bridges AJ, Graziano FM, Calhoun W, Reizner GT. Hyperpigmentation, neutrophilic alveolitis and erythema nodopigmentation, neutrophilic alveolitis and erythema nodosum resulting from

7 Gorard DA. Late onset drug fever associated with minocycline. Postgrad Med f 1990;66:404-5.

8 Sitbon O, Bidel N, Dussopt C, Azarian R, Braud ML, Lebargy $\mathrm{F}$, et al. Minocycline pneumonitis and eosinophilia. A report on eight patients. Arch Intern Med 1994;154:1633-40.

9 Gough A, Chapman S, Wagstaff K, Emery P, Elias E. Minocycline induced autoimmune hepatitis and systemic lupus erythematosus-like syndrome. BMF 1996;312:169-72.

10 Puyana J, Urena V, Quirce S, Fernandez-Rivas M, Cuevas M, Fraj J. Serum sickness like syndrome associated with minocycline therapy. Allergy 1990;45:313-5.

11 Byrne PA, Williams BD, Pritchard MH. Minocycline related lupus. Br $₹$ Rheumatol 1994;33:674-6.

12 Kallenber CGM, Brouwer F, Weening J, Cohen Tervaert JW. Antineutrophilic cytoplasmatic antibodies: current diagnostic and pathophysiological potential. Kidney Int 1994;46:1-15.

13 Wiik A, Stummann L, Kjeldsen L, Borregaard N, Ullman S, Jacobsen $S$, et al. The diversity of perinuclear antineutrophilic cytoplasmatic antibodies (pANCA) antigens. Clin Exp Immunol 1995;101:15-17.
14 Gross WL, Schmitt WH, Csernak E. ANCA and associated diseases: immunodiagnostic and pathogenetic aspects. Clin Exp Immunol 1993;91:1-12.

15 Wiik A. Granulocyte specific antinuclear antibody. Allergy 1980;35:263-89.

16 Nassberger L, Sjoholm AC, Jonsson H, Sturfel G, Akesson A. Autoantibodies against neutrophilic cytoplasmatic components in SLE and in hydralazine lupus. Clin Exp components in SLE and

17 Dolman KM, Gan RO, Verat TJ, Zevenberger G, Maingay D, Nikkels RF, et al. Vasculitis and antineutrophilic cytoplasmatic autoantibodies associated with propylthiouracil therapy. Lancet 1993;342:651-2.

18 Jones BF, Major GAS. Crescentic glomerulonephritis in a patient taking penicillamine associated with antineutrophil cytoplasmatic antibody. Clin Nephrol 1992;38:293.

19 Cambridge G, Wallace H, Bernstein RM, Leaker B. Autoantibodies to myeloperoxidase in idiopathic and drug induced systemic lupus erythematosus and vasculitis. $\mathrm{Br} \mathcal{F}$ Rheumatol 1994;33:109-14.

20 D'Cruz D, Chesser AMS, Lightowler C, Comer M, Hurst MJ, Baker LRI, et al. Antineutrophil cytoplasmatic antibody positive crescentic glomerulonephritis associated with antithyroid drug treatment. $\mathrm{Br} \mathcal{F}$ Rheumatol 1995; 34:1090-1.

21 Singer S, Parry RG, Deodhar HA, Barnes JN. Acute interstitial nephritis, omeprazole and antineutrophilic cytoplasmatic antibodies. Clin Nephrol 1994;42:280.

22 Yamamoto K, Kawanishi S. Free radical production and site-specific DNA damage induced by hydralazine in the presence of metal ions of peroxidase/hydrogen peroxide. Biochem Pharmacol 1991;41:905-13.

23 Hofstra AH, Li-Muller SMA, Uetrecht JP. Metabolism of isoniazid by activated leucocytes: possible role in drug induced lupus. Drug Metab Dispos 1992;20:205-10.

24 Jiang X, Khursigara G, Rubin RL. Transformation of lupusinducing drugs to cytotoxic products by activated neutrophils. Science 1994;266:810-3. 